Retrospective Review

\title{
Low Volume Neurolytic Retrocrural Celiac Plexus Block for Visceral Cancer Pain: Retrospective Review of 507 Patients with Severe Malignancy Related Pain Due to Primary Abdominal Cancer or Metastatic Disease
}

\author{
Abed Rahman, MD¹, Raed Rahman, DO ${ }^{1}$, George Macrinici, MD¹, and Sam Li, MD²
}

From: 'Interventional Pain Management, Department of

Pain Medicine, Midwestern Regional Medical Center, Zion, IL; ${ }^{2}$ Department of Anesthesiology and Pain Management, JHS Hospital of Cook County, Chicago, IL

Address Correspondence: Abed Rahman, MD Interventional Pain Management, Pain Management Physician, Department of Pain Medicine, Midwestern Regional Medical Center, 2520 Elisha Avenue, Zion, IL 60099

E-mail:

Abed.rahman@ctca-hope.com

Disclaimer: There was no external funding in the preparation of this manuscript. Conflict of interest: Each author certifies that he or she, or a member of his or her immediate family, has no commercial association (i.e., consultancies, stock ownership, equity interest, patent/licensing arrangements, etc.) that might pose a conflict of interest in connection with the submitted manuscript.

Manuscript received: 11-21-2017 Revised manuscript received: 03-09-2018 Accepted for publication: 03-26-2018

Free full manuscript: www.painphysicianjournal.com
Background: Abdominal pain from primary cancer or metastatic disease is a significant cause of pain for patients undergoing treatment for the disease. Patient's pain may be resistant to conventional analgesics. The need for timely pain relief in order to facilitate further care in the cancer treatment plan should be a priority.

Objectives: The aim of this retrospective observational review was to assess the relief given with a low volume neurolytic retrocrural celiac plexus nerve block, the duration of the procedure, the duration of relief, the reduction in daily opioid consumption, and the improvement of quality of life in a patient suffering from incapacitating abdominal pain due to primary abdominal malignancy or abdominal metastatic disease. Patients were given a neurolytic celiac plexus block without previous diagnostic block due to multiple comorbidities.

Study Design: This is a retrospective, observational study.

Methods: Five hundred and seven patients were studied and data at 5 months for 455 patients were retained at the end of the review. They were evaluated in the pain center prior to and after the neurolytic retrocrural celiac plexus nerve block under fluoroscopic guidance. They were assessed on duration of procedure, pain scores (numeric rating scale $0-10$ ), daily opioid consumption, quality of life improvement (simple yes or no question at 3 months) and routine follow-up during treatment for the cancer for 6 months or end of life. All data was gathered by extensive chart review and placed on a spreadsheet for analysis.

Results: Follow-up was completed 6 months after the procedure. Pain scores, daily opioid consumption, and quality of life showed improvement for the duration of the study. There was some return in pain during the fourth to sixth month due to disease progression and the anticipated duration of the neurolytic agent. Some short duration known side effects did occur. An initial vascular contrast uptake of $6.7 \%$ was noted during the procedure while utilizing digital subtraction angiography with fluoroscopy.

Limitations: A larger sample size would be ideal, as well as, a prospective trial with a control group, but this is unrealistic in our patient population. A proven quality of life questionnaire would be beneficial. Comparing alcohol, phenol and radiofrequency thermocoagulation would be interesting to equate duration, effect, and side effects.

Conclusion: Low volume neurolytic retrocrural celiac plexus nerve block with phenol is a safe procedure providing up to 6 months of pain relief and is an effective, well-established, minimally time-consuming procedure for abdominal pain due to primary malignancy or metastatic spread.

Key words: Celiac plexus, neurolytic, abdominal cancer pain, pain, retrocrural, cancer pain

Pain Physician 2018: 21:497-504 
ancer-related pain from either primary abdominal malignancies or metastatic spread may be a source of significant pain often resistant to conventional analgesic and standard therapy. Typical treatment of cancer-related pain often relies heavily on a combination of narcotic(s) and nonnarcotic adjuvants (1-3). During this tumultuous time, patients deal with multiple stressors (e.g., emotional and physiological). Pain remains a limiting factor to both function and quality of life (QOL) in these patients. Initial evaluation and treatment should focus on achieving pain relief, return of acceptable function, and the ability to perform activities of daily living (ADLs) (4,5).

For patients with upper abdominal pain secondary to malignancy, the celiac plexus nerve block (CPNB) has been used to manage pain located to the epigastric, periumbilical, left upper quadrant, right upper quadrant and mid thoracic region (6-8). The celiac plexus contains visceral afferent and efferent which provides sensation and autonomic innervation to a majority of the upper abdominal region. Preganglionic fibers from vertebral levels T5-T12 exit the ventral roots and travel caudally as the greater (T5-9), lesser (T10-11), and least (T12) splanchnic to coalesce anterolateral to the aorta at the level between T12-L1 intervertebral disc and L2 vertebral body to form the celiac plexus $(9,10)$.

With better understanding of the anatomy, there have been several approaches to the block (e.g., anterior, posterior, antecrural, retrocrural, transaortic, transgastric) developed to assess this region for denervation. In addition, there have been various methods to achieve denervation (e.g., radiofrequency ablation and chemical neurolysis with alcohol or phenol) of the celiac plexus $(3,6,11-13)$. Given the number of different approaches and modalities of performing chemical or thermal ablation of the celiac plexus, there are global complications related to the procedure and complications specific to the approach. The most common complication related to the procedure is transient hypotension; whereas, paralysis, although very rare, is the gravest reported complication (14-16). With multiple techniques and limited cross-comparison analysis, there has yet to be determined a single best technique and/or approach. Additionally, with the advent of newer techniques, often the more traditional ones are dismissed or simply forgotten. However, when performing a CPNB, utilization of the best method and/or approach can be measured in quality and duration of pain relief, a reduction in daily opioid consumption, procedural duration and complications. It is also noteworthy to consider any distortion to a patient's abdominal anatomy secondary to malignancy as it may limit a provider's access via a specific approach (17). The purpose of this study is to demonstrate the effectiveness of the neurolytic celiac plexus block with low volumes, short duration, done early in the treatment period to facilitate pain relief without complications on the frail cancer patient.

This study is also to assess the efficacy of low volume neurolytic retrocrural celiac plexus nerve block (NRCPB) in the management of upper abdominal cancer-related pain. Value is measured based on the reduction in patient pain score, a reduction of daily opioid consumption, an improvement in quality of life (QoL), procedural time, and related complications. We avoided performing a diagnostic block on this patient population due to multiple comorbidities and risk of repeat procedures and repetitive anesthetic exposure.

\section{Methods}

This is a retrospective, observational study of patients evaluated and treated at the Cancer Treatment Center of America (a hospital-based procedure center located at the Midwestern Regional Medical Center in Zion, IL) from Sept. 12, 2011 to Dec. 31, 2015. The patient list was generated using the pain center case log. This study was approved by the institutional review board for the retrospective gathering and publishing of relevant data. The data was collected by 2 principal investigators and the results were reviewed by an independent statistician. All authors had full access to the data. There was no sponsor for the review or financial incentive at any point of the data-gathering process. All the authors reviewed and provided feedback on all subsequent manuscript drafts. All the authors vouch for the completeness and accuracy of the data analyses and affirm that the retrospective data collection was conducted and reported with fidelity to the institutional review board. Review of the patient records included the routine details on all patients in the current pain center prior to and following any pain procedure with a standard, detailed questioning and charting by the pain physicians, nurse practitioners, physician assistants and nurses. The standard, detailed data gathering was collected by the pain service and tabulated on an Excel spreadsheet for the study. Procedures were done in the hospital surgical operating room or interventional radiology suites room under local, moderate sedation or monitored anesthesia care.

Patients reviewed in the study suffered from 
severe, incapacitating abdominal pain, some with midthoracic back pain from primary malignancy or metastatic disease. Patients included were those unable to achieve satisfactory pain relief with pharmacological agents including escalating oral narcotics, transdermal opioids, intravenous opioids, sublingual opioids, neoadjuvant therapy, and complementary and alternative medicine. Patients selected for the study receive a pain score of $<5$ using a 0-10 scale, or those who experienced adverse reactions to medications. During initial history and physical examination, an extensive evaluation was completed with the patients and caregivers. The patient's subjective pain description, location, radiation and aggravating factors were detailed for procedure purposes to determine if the block was appropriate (18). Exclusion criteria included contraindications for regional blockade such as coagulopathy, infection at entry point, and/or sepsis, abdominal pain due to ascites, carcinomatosis, and altered mental status.

Pain scores were recorded using the numerical rating scale (ranging from 0-10) during each patient encounter and immediately after procedure completion. The QoL was a yes or no question (Are you happy with your quality of life after the block?) in which the patient would answer. Opioids were converted to oral morphine equivalents for a total of a 24-hour consumption rate to standardize the opioid dosing discussion (19). Total daily opioid doses were collected and entered by the pain nurses. Assessment of the various markers were preoperative, postoperative, 1-week post-operative, and 1-, 2-, 3-, 4-, 5-, and 6-months post-procedure or end of patients' life, which is routine data in our pain center.

Prior to the start of each procedure, informed consent was obtained for each patient, as well as, each patient receiving intravenous fluid hydration with 1-2 liters of balanced salt solution. The retrocrural celiac plexus approach was done at the L1 level with aim toward T12. Anterior and posterior radiographic imaging aligning the spinous process of the T12 and L1 junction was used, along with 15-20-degree oblique rotation and cephalad orientation to move the transverse process from the center of the vertebral body. Local anesthetic $1 \%$ lidocaine with sodium bicarbonate 20 is infiltrated along the injection direction. The anesthetic of choice was started with the patient remaining communicative and responses deemed appropriate during the injection of the local test dose and neurolytic portion. A 22-gauge or 25-gauge, 3.5 inch to 7-inch curved spinal needle (depending on patient's habitus) was advanced until the periosteal surface of the L1 vertebral body is contacted utilizing multiple AP, lateral and oblique views. In the lateral view, the spinal needle was advanced to the anterior border of the T12 L1 level (Figs. 1A and B).

Contrast dye studies were done to verify spread and location. No air bubbles in the extension tubing were present at any time to avoid potential air embolus. Digital subtraction angiography was utilized to verify any intravascular uptake or abnormal spread patterns. A test dose of $1 \mathrm{~mL}$ of $0.5 \%$ bupivacaine with 1: 200,000 epinephrine per site was given. After no signs of vascular uptake, $3-5 \mathrm{~mL}$ of $6 \%$ aqueous phenol was injected in $1 \mathrm{~mL}$ aliquots while speaking to patient and the needle was removed after being flushed with local anesthetic. The procedure time averaged 16.3 minutes with minimal anesthetic or no sedation, with the patient remaining communicative during the key portions of the procedure. The patient would then be placed on the transport stretcher and remain prone for 30 minutes to avoid neuroforaminal spread. Patients were monitored in recovery for 1 hour for adverse events, the ability to eat, and to void easily. They were discharged once the hospital post anesthesia care discharge criteria were met. Patient were usually discharged home the same day with a caregiver and called within 24 hours.

\section{RESULTS}

The study included 507 patients, all of which had suffered severe epigastric pain, right or left upper quadrant pain, flank pain or thoracic pain. All patients were $>18$ years of age, ranging from 19-73 with a median age of 57.3. Male and female patients were included with $52.7 \%$ being female (267 out of 507). All patients had failed conservative therapies including escalating large doses of opioid, nonnarcotics, complementary and alternative medicines, acupuncture, mind-body medicine, and physical therapy $(21,22)$. All the patients were in various stages of cancer and in progression states and had uncontrolled pain. The cause of the abdominal pain was primary abdominal malignancy such as, pancreatic, gastric, colon, renal, or distant primary malignancy with metastasis to the abdomen. Main indications for the procedure focused on the patient's subjective description of pain location, e.g., the epigastric, periumbilical, upper abdominal quadrants, flanks and mid-thoracic region (18). Nonmalignant and functional causes of abdominal pain were ruled out by primary service prior to the intervention. The intraoperative period was uneventful with 


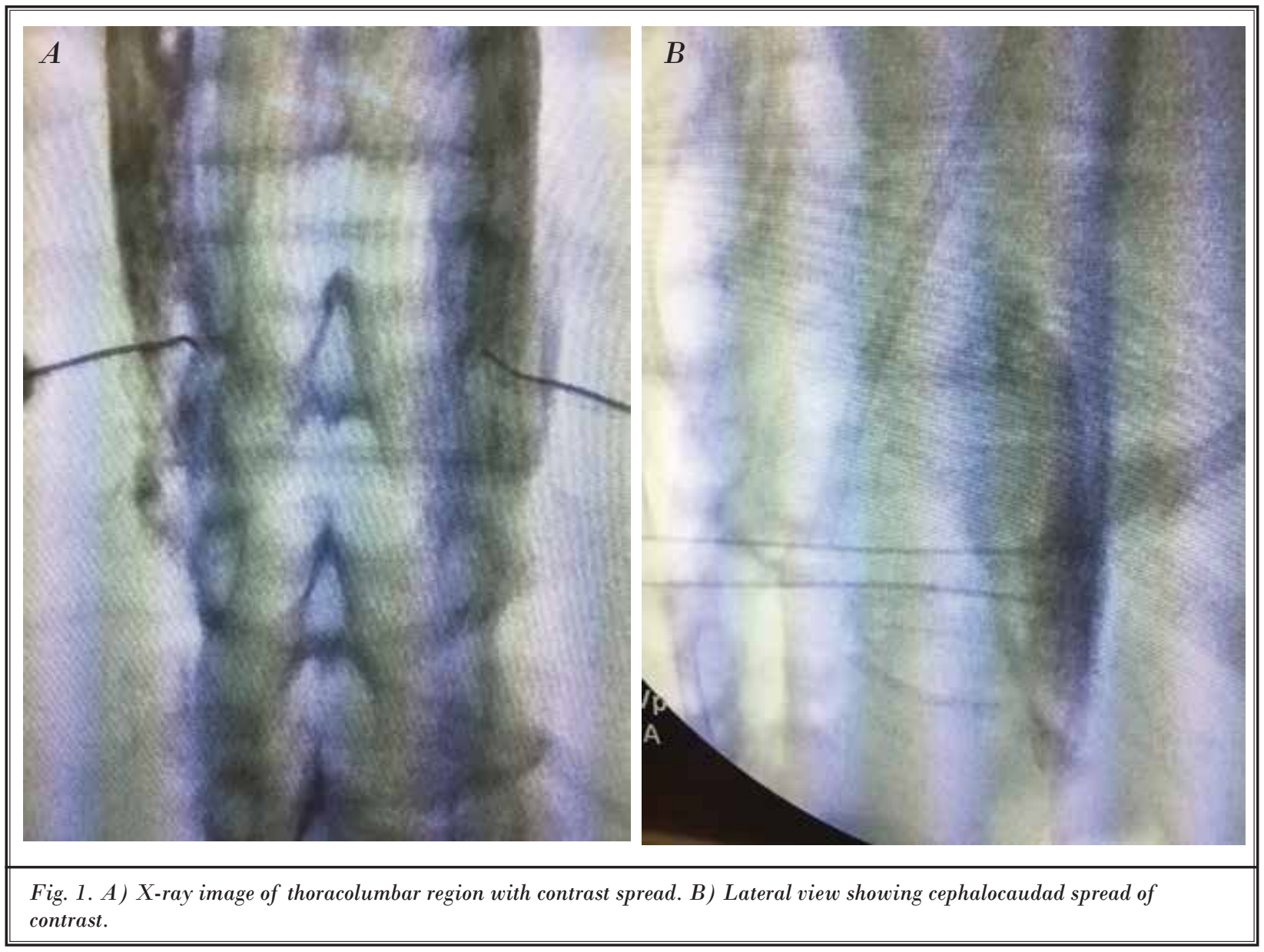

expected intraoperative decrease $(28 \%, 142$ out of 507 patients) in mean arterial blood pressure $>20 \%$ from baseline, responding to intravenous fluid therapy. All patients tolerated the procedure well, without any serious adverse events or complaints of an increase in pain score. The average procedure duration was 16.3 minutes (9.4 - 19.5 minutes). The post-operative period was uneventful with no significant procedure related events. The follow-up period was 6 months post-procedurally or end of life.

Data from 507 patients regarding pain and morphine equivalents consumption were collected and analyzed in this retrospective study. Pain scores and morphine equivalents consumptions were recorded at pre-op and post-op, as well as after 1 week, and then every month until the end of the 5 month or patient death. The number of records at each time point is listed in Table 1. Collected data was sent to an independent statistician for review and analysis.
The mean pain score of pre-op was 8.48. Mean pain scores were reduced after the procedure and during the follow-ups (Table 2, Fig. 2). Pain at post-op and followups was significantly reduced compared to pre-op, $P<$ 0.001, Wilcoxon signed-rank test (Table 2, Fig. 3).

The pre-op mean morphine consumption was $125.27 \mathrm{mg} / 24$ hours. Mean morphine consumption was reduced during the follow-up (Table 3 ). The reduction rates of morphine consumption at follow-ups was significant compared to pre-op, $P<0.001$, Wilcoxon signed-rank test (Fig.4).

Adjuvants of pain continued and were adjusted as needed. Those included, pregabalin, gabapentin, duloxetine, nortriptyline, amitriptyline, NSAIDS, and muscle relaxants $(23,24)$. As with all patients in the center, patients were encouraged to participate in mindbody medicine and acupuncture to assist with pain and coping mechanisms (25).

The QoL was a simple yes or no question to assess 
improvement of patient or caregivers' perspective after the neurolytic block, which was documented in the medical record on the 3-month visit. The document responses were included in the medical record (Table 3).

Complications and expected side effects included transient diarrhea in 77 patients $(15.1 \%)$ lasting up to 2 weeks. One patient did have orthostatic hypotension with postural effects lasting 4 weeks and was required to see a cardiologist for a blood pressure medication regimen adjustment. Of the patients undergoing the procedure $6.7 \%$ (34 out of 507) were noted to have initial venous and arterial contrast uptake on digital subtraction angiography (DSA). Despite a negative aspiration, needles were redirected until angiogram showed no vascular uptake.

\section{Discussion}

This study demonstrates the benefit of a low volume NRCPB in the treatment of primary or metastatic pain involving the epigastric, periumbilical, upper abdominal quadrants, flanks and mid-thoracic region. Low volume NRCPB is a proven safe and efficacious therapy for patients who have already failed to receive adequate pain control despite maximal medical therapy. In addition, this retrospective study, not only, demonstrates lasting benefits up to 5 months (the measured end-point of the study), but also, an improvement in QoL and reduction in opioid consumption. This work adds to the cancer pain management literature in that it advocates for the consideration of a retrocrural blockade early on in cancer treatment for any malignancy-related visceral abdominal pain.

Based on previous studies, side effects such as orthostatic hypotension and transient diarrhea are well-known, and anticipated effects of the blocks and may occur in approximately $38 \%$ to $44 \%$

Table 1. Number of records.

\begin{tabular}{|l|c|c|c|c|c|c|c|c||}
\hline & Pre-op & Post op & 1 wk & 1 mo & 2 mo & 3 mo & 4 mo & 5 mo \\
\hline Pain & 507 & 507 & 507 & 497 & 487 & 476 & 474 & 456 \\
\hline Morphine & 507 & NA & NA & 497 & 487 & 477 & 474 & 455 \\
\hline
\end{tabular}

Table 2. Mean pain reduction from pre-op.

\begin{tabular}{|l|c|c|c|c|c|c|c|}
\hline & Post op & $\mathbf{1}$ wk & $\mathbf{1}$ mo & $\mathbf{2}$ mo & $\mathbf{3}$ mo & 4 mo & $\mathbf{5}$ mo \\
\hline Pain reduction & 7.32 & 5.73 & 6.52 & 6.63 & 6.34 & 5.62 & 4.81 \\
\hline
\end{tabular}

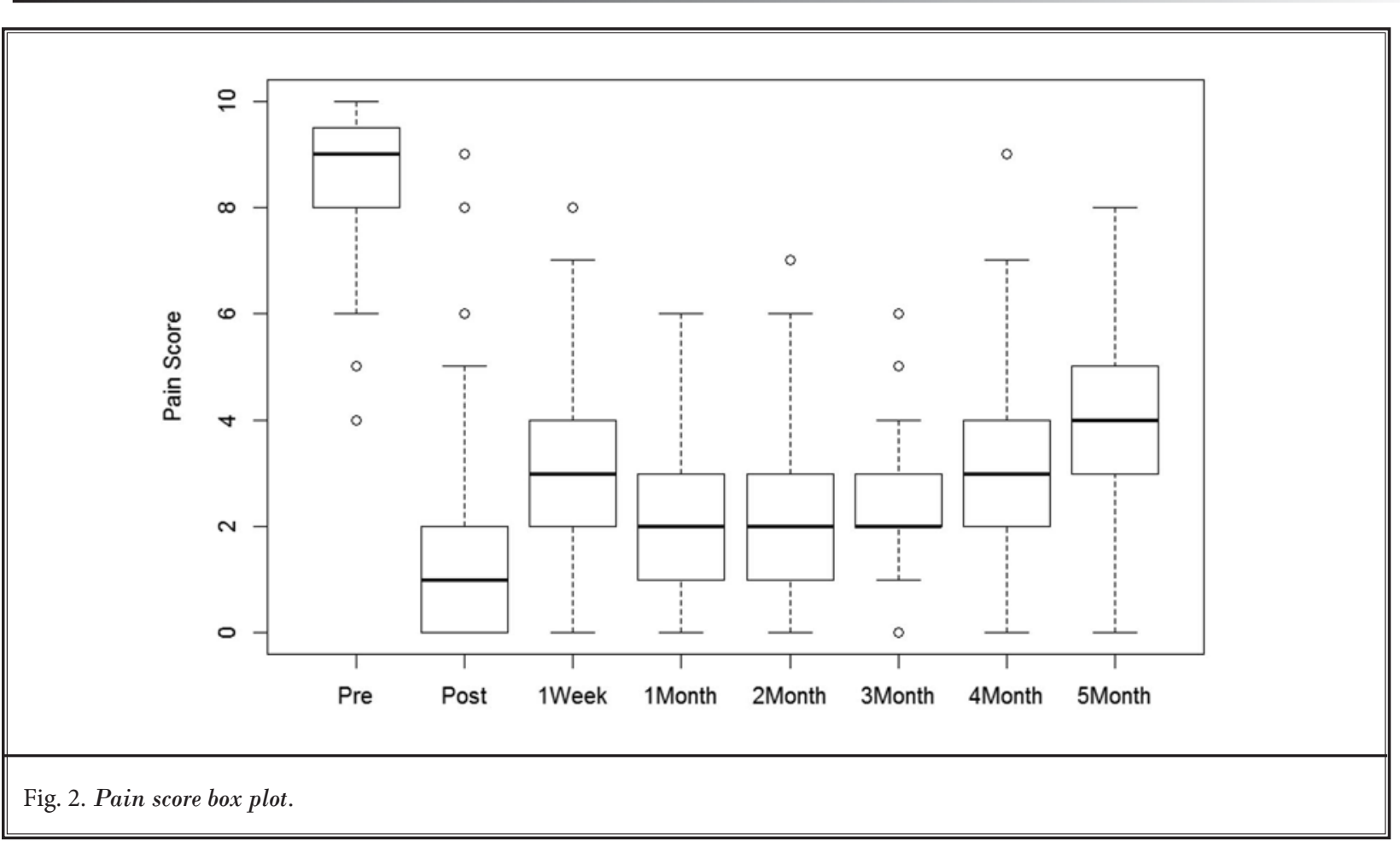



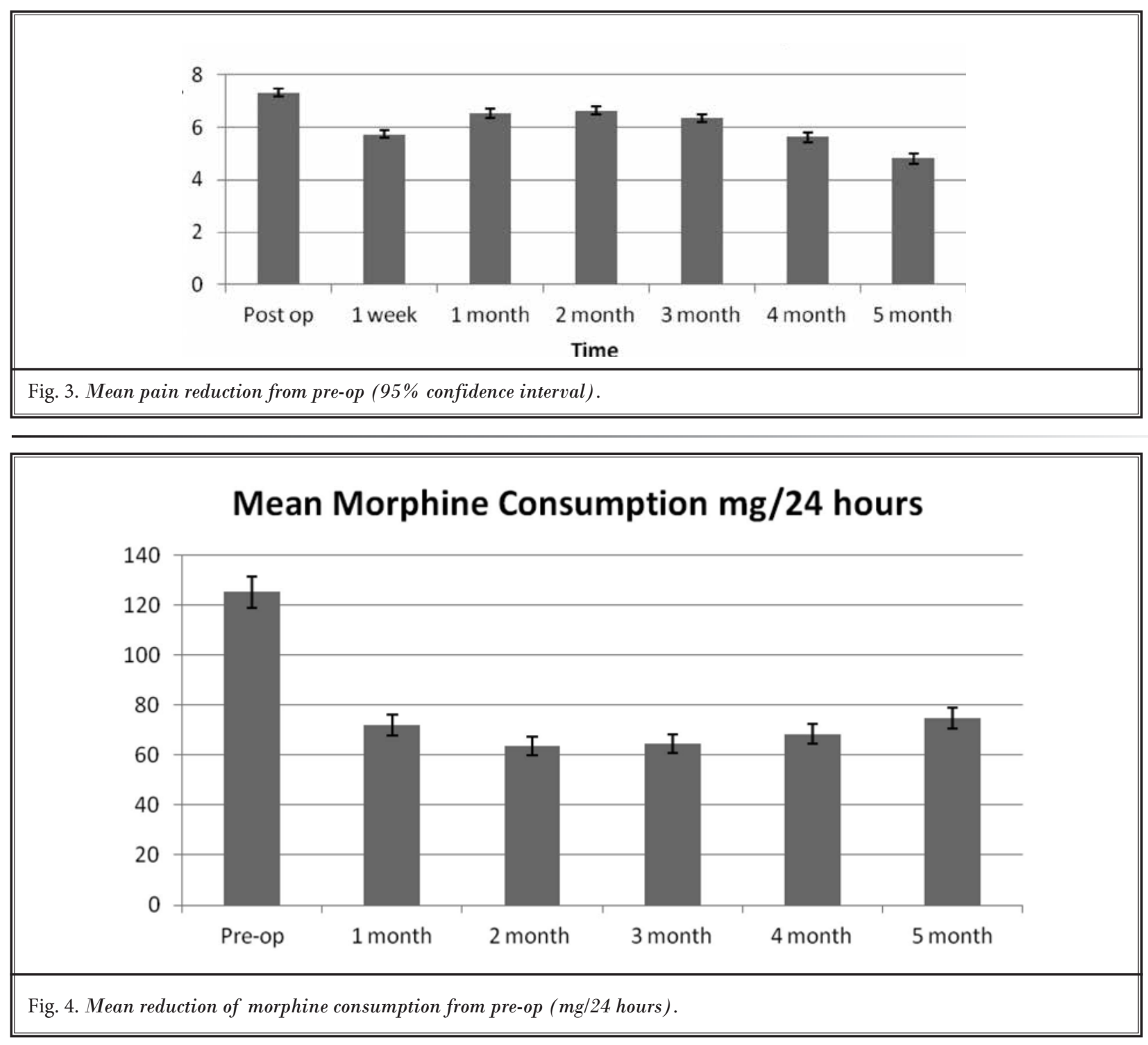

Table 3. Documented chart of responses for quality of life improvement at 3 months (388 of 507).

\begin{tabular}{|c|c|}
\hline Yes & No \\
\hline 388 & 119 \\
\hline $76.5 \%$ & $23.5 \%$ \\
\hline
\end{tabular}

of patients respectively. These percentages were calculated based on a meta-analysis of celiac plexus blocks independent of the approach. In comparison, this study was done via a retrocrural approach, which reported a complication rate of $2 \%$ and $15 \%$ for orthostatic hypotension and transient diarrhea, respectively. With diligent utilization of the fluoroscopy C-arm, digital subtraction angiography (DSA) in all procedures performed, $6.7 \%$ of our procedures were positive for contrast venous and arterial uptake requiring repositioning, but no symptoms of phenol toxicity (e.g., tremors and seizures) and/or paraplegia were recorded. There were no occurrences of other rare complications such as retroperitoneal hematoma, pneumothorax, cardiac arrest and renal/intestinal injury. Given the relatively low risk to benefit ratio, and analgesic benefit, it is the authors' belief that this modality of pain therapy should be offered as an initial option for patients suffering from abdominal malignant pain.

Relative to past studies $(6,11)$, this retrospective analysis encompasses a larger study size of 507 pa- 
tients limited to 2 principal investigators to maintain a homogenous technical approach and limit inter-user variability. Additionally, average procedural time was 16.3 minutes (9.4 - 19.5 mins) and overall reported complication rates were low, highlighting the investigators familiarity with NRCPB. Each patient was personally seen and offered the procedure by the principal investigators at The Cancer Treatment Centers of America, Midwestern Regional Medical Center in Zion, IL. Specific to this study, the inclusion criteria require patients to have already demonstrated failure of adequate pain control with maximal medical therapy. Therefore, a strong correlation can be inferred between low volume NRCPB and the reduction in patient's pain score, as well as, morphine equivalent usage and an improvement in QoL.

Although, a retrospective study does not carry as much empirical evidence as a properly conducted randomized, controlled trial, and we understand and agree with the limitations of this study type. For future studies, we suggest a crossover, randomized, controlled trial, but this is not practical in our patient population, in which both prongs of the study eventually received interventions at staggered times. Another limitation found in this study, and what we recommend being utilized in future studies, is a more in-depth questionnaire of QOL (e.g., SF-36) given the basic yes or no question routinely asked on follow-up visits. Pain scores and morphine equivalents consumptions were recorded at pre-op and post-op, as well as after 1 week, and then every month until the end of the 5 month or patient death. We propose that future studies maintain periodic correspondence with patients until end of life to fully appreciate the effect of low volume NRCPB. Cancerrelated abdominal pain remains difficult to treat and taxing, both emotionally and physically, on the patient and their support system. High dose opioid and adju- vant therapy are often partially effective and limited by side effects and patient compliance. We demonstrate the need for early referral for these cancer patients to assist with pain control to facilitate therapies to the underlying malignancy. This technique, given the short duration of procedure time, low volume of phenol and quick recovery, demonstrates a reasonable technique to assist our patients, as well as, helping to avoid a second diagnostic block procedure. This block may be effectively utilized for multiple types of abdominal pain from primary organ cancer to metastatic disease and should not be limited to pancreatic cancer. In addition, the ability to repeat this block and avoid implantation of an intrathecal pain pump early on allows for quicker recovery and shorter procedural times. While there is debate on whether to perform a diagnostic block or proceed directly with a NRCPB, we chose to proceed directly with NRCPB to avoid exposing patients to repeat procedures and multiple anesthetic exposures in their frail state (26). This block may enhance the QoL of the patient and promote an improvement in functionality. With celiac plexus blocks and splanchnic nerve blocks being clinically comparable, it is the authors training and appreciation of anatomical changes due to malignancy that make low volume NRCPB the preferred technique (27).

\section{Conclusion}

A low volume NRCPB provides a safe and efficacious therapy in the armamentarium of an interventional pain specialist. Low volume NRCPB has proven benefit in QOL, pain score, and opioid consumption and provides long-lasting relief concordant to these patient's terminal prognosis. Given this patient population, proceeding directly to a neurolytic block to avoid repeated procedures and anesthetics is reasonable. 


\section{References}

1. Ventafridda $\mathrm{V}$, Tamburini M, Caraceni A, De Conno F, Naldi F. A validation study of the WHO method for cancer pain relief. Cancer 1987; 59:850-856.

2. American Cancer Society. Cancer Facts \& Figures 2015. American Cancer Society. https://www.cancer.org

3. Tewari S, Agarwal A, Dhiraaj S, Gautam SK, Khuba S, Madabushi R, Shamshery C, Kumar S. Comparative evaluation of retrocrural versus transaortic neurolytic celiac plexus block for pain relief in patients with upper abdominal malignancy: A retrospective observational study. Indian J Palliat Care 2016; 22:301-306.

4. Bjelic-Radisic V, Jensen PT, Vlasic KK, Waldenstrom AC, Singer S, Chie W, Nordin A, Greimel E. Quality of life characteristics inpatients with cervical cancer. Eur J Cancer 2012; 48:3009-3018.

5. Bhaskar AK. Interventional management of cancer pain. Curr Opin Support Palliat Care 2012; 6:1-9.

6. Papadopoulos D, Kostopanagiotou G, Batistaki C. Bilateral thoracic splanchnic nerve radiofrequency thermocoagulation for the management of end-stage pancreatic abdominal cancer pain. Pain Physician 2013; 16:125-133.

7. Loukas M, Klaassen Z, Merbs W, Tubbs RS, Gielecki J, Zurada A. A review of the thoracic splanchnic nerves and celiac ganglia. Clin Anat 2010; 23:512-522.

8. Raj P, Rauck R, Racz G. Autonomic nerve blocks. In: Raj P, editor. Pain Medicine: A Comprehensive Review. Mosby, St Louis, MO, 1996, pp. 227-256.

9. Yang HJ, Gil YC, Lee WJ, Kim TJ, Lee HY. Anatomy of thoracic splanchnic nerves for surgical resection. Clin Anat 2008; 21:171-177.

10. Gong H, Ma R, Gong J, Cai C, Song Z, $\mathrm{Xu}$ B. Distal pancreatectomy with en bloc celiac axis resection for locally ad- vanced pancreatic cancer: A systematic review and meta-analysis. Medicine (Baltimore) 2016; 95:e3061.

11. Koyyalagunta D, Engle MP, Yu J, Feng L, Novy DM. The effectiveness of alcohol versus phenol based splanchnic nerve neurolysis for the treatment of intra-abdominal cancer pain. Pain Physician 2016; 19:281-292.

12. Ischia S, Ischia A, Polati E, Finco G. Three posterior percutaneous celiac plexus block techniques. A prospective, randomized study in 61 patients with pancreatic cancer pain. Anesthesiology 1992; 76:534-540.

13. Kamdar MM, Edwards DA, Thabet AM, Volney SJ, Rathmell JP. A novel modified retrocrural approach for celiac plexus block: The single-needle retroaortic technique. Reg Anesth Pain Med 2015; 40:610-615.

14. McAninch SA, Raizada MS, Kelly SM. Pulmonary embolism following celiac plexus block and neurolysis. Proc (Bayl Univ Med Cent) 2016; 29:329-330.

15. Wong GY, Brown DL. Transient paraplegia following alcohol celiac plexus block. Reg Anesth 1995; 20:352-355.

16. Buscher HC, Lenders JW, Wilder-Smith $\mathrm{OH}$, Sweep CG, van Goor H. Bilateral thoracoscopic splanchnicectomy for pain in patients with chronic pancreatitis impairs adrenomedullary but not noradrenergic sympathetic function. Surg Endosc 2012; 26:2183-2188.

17. Elahi F, Wu WY, Callahan D, Bhandary AK, Beutler BC, Lassalle CA. Images in anesthesiology: Reversible anterior spinal artery syndrome during celiac plexus block. Anesthesiology 2013; 118:187.

18. Novy DM, Engle MP, Lai EA, Cook C, Martin EC, Trahan L, Yu J, Koyyalagunta D. Effectiveness of splanchnic nerve neurolysis for targeting location of cancer pain: Using the pain drawing as an outcome variable. Pain Physician 2016; 19:397-403.

19. Best CA, Best AA, Best TJ, Hamilton DA. Buffered lidocaine and bupivacaine mixture - the ideal local anesthetic solution? Plast Surg (Oakv) 2015; 23:87-90.

20. Lahoud MJ, Kourie HR, Antoun J, El Osta L, Ghosn M. Road map for pain management in pancreatic cancer: A review. World J Gastrointest Oncol 2016; 8:599-606.

21. Kilian-Kita A, Puskulluoglu M, Konopka K, Krzemieniecki K. Acupuncture: could it become everyday practice in oncology? Contemp Oncol (Pozn) 2016; 20:119-123.

22. Yajima R, Matsumoto K, Ise $Y$, Suzuki N, Yokoyama Y, Kizu J, Katayama S. Pregabalin prescription for terminally ill cancer patients receiving specialist palliative care in an acute hospital. J Pharm Health Care Sci 2016; 2:29.

23. Nurwidya F, Syahruddin E, Yunus F. Pain management in lung cancer. Adv Respir Med 2016; 84:331-336.

24. Oh B, Eade T, Kneebone A, Pavlakis N, Clarke S, Eslick G, River J, Back M. Factors affecting whether or not cancer patients consider using acupuncture. Acupunct Med 2017; 35:107-113.

25. Vorenkamp K, Dahle N. Diagnostic celiac plexus block and outcome with neurolysis. Techniques in Regional Anesthesia and Pain Management 2011; 15:28-32.

26. Shwita AH, Amr YM, Okab MI. Comparative study of the effects of the retrocrural celiac plexus block versus splanchnic nerve block, C-arm guided, for upper gastrointestinal tract tumors on pain relief and the quality of life at a six-month follow up. Korean J Pain 2015; 28:22-31. 